\title{
USING STATISTICAL METHODS TO EVALUATE SOME INBRED LINES OF MAIZE UNDER DIFFERENT SOWING DATE \\ Hoda E.A. Ibrahim ${ }^{1}$ and M.M.M Hassan ${ }^{2}$ \\ 1- Center Lab. For Design and Stat. Anal. Res., ARC, Giza. Egypt. \\ 2- Field crops Res. Institute, ARC and Giza. Egypt.
}

\section{ABSTRACT}

GGE biplot analysis is an effective method which is based on the principal component analysis (PCA) in order to fully evaluate multi environmental yield trials (METs). Additive main effects and multiplicative interaction (AMMI) is an alternative method for assessing phenotypic stability and adaptability. In this research, data of 16 maize inbred lines were used to perform AMMI and GGE biplot analysis. These inbred lines were evaluated under four sowing dates during two successive summer seasons of 2012 and 2013. Inbred line by sowing dates table was used for performing the analysis. Based on both mean grain yield and yield stability, inbred lines Giza 603 , Sids 34, Giza 629 and Giza 628 proved to be superior and also had greater mean performance among the test inbred lines. Graphic analysis was used to identify the most suitable inbred lines for each tested sowing date. Inbred lines Sids 34, Giza 629, Gemmeiza 1021, Sids 7, Giza 612, Giza 628, Giza 603, Gemmeiza 1004 and Giza 602 were identified as suitable in all sowing dates. The AMMI analysis identified the best sowing date as second sowing date D2 which had the highest PCA1 and the best 1PCA2 values. The GGE biplot graphics revealed four sowing dates were clustered into two groups in all cases Group one included sowing date 1 and 2 Group two included sowing date 3 and 4, inbred line Giza 603the best performer in all inbred lines, followed by Sids 34, Giza 629 and Giza 628.

Inbred lines Giza 603, Sids 34, Giza 628, had the highest yield stability into the best sowing dates. Thus sowing date 1 and 4 are the most discriminative sowing dates. . This inbred lines intervention in the production of many commercial hybrids.

Keywords: maize, GGE biplot, AMMI, PCA, G $\times$ E interaction, sowing dates.

\section{INTRODUCTION}

Maize (Zea mays L.), one of the major field crops in Egypt. Phenotype is a combination of genotype $(G)$, environment $(E)$ and genotype $\times$ environment interaction $(\mathrm{G} \times \mathrm{E}) . \mathrm{G} \times \mathrm{E}$ usually complicates the process of selecting superior genotypes.

Consequently, multi-environment trials (METs) are widely used by plant breeders for evaluating the relative performance of genotypes over the target environments (Delacy et al., 1996). Additive main effects and multiplicative interaction (AMMI,Gauch, 1992) method is commonly used to analyze MET data and have also been applied in $\mathrm{G} \times \mathrm{E}$ interaction studies in maize and other crops (Bertoia et al., 2006; Lee et al., 2003; Ades and Garnier-Géré, 1997; Wu and Matheson, 2005; Butrón et al., 2004).

The GGE biplot analysis of these data showed that ideal test environments could discriminate superior performing maize from poor ones, and identified four-environments in the target areas. GGE biplot analysis was recently developed to simultaneously use some of the functions of these methods. In phenotypic variation, $E$ explains most of the variation, and $G$ and $G \times E$ are usually small (Yan, 2002). However, only $G$ and $G \times E$ interaction are relevant to cultivar evaluation, particularly when $G \times E$ interaction is 
determined as repeatable (Hammer and Cooper, 1996). Hence, Yan et al. (2000) deliberately put the two together and referred to the combination as GGE. following the proposal of Gabriel (1971), the biplot technique was also used to display the GGE of MET data, and is referred to as a GGE biplot (Yan, 2001; Yan et al., 2000). The GGE biplot is in fact a data visualization tool that graphically displays $G \times E$ interaction in a two way table (Yan et al., 2000). The GGE biplot is an effective tool for the following applications 1. Genotype evaluation (mean performance and stability), and 2. Environmental evaluation (to discriminate among genotypes in target environments). GGE biplot analysis is increasingly being used in $\mathrm{G} \times \mathrm{E}$ interaction studies in plant breeding research (Butron et al., 2004; Dehghani et al., 2006; Kaya et al., 2006; Samonte et al., 2005; Yan and Tinker, 2005). AMMI is a multivariate technique for assessinglthe phenotypic stability and adaptability of genotypes (Pacheco and Vencovsky, 2005). This method partitions the overall variation into $G, E$ and $G \times E$. The data structure that $A M M I$ and $G G E$ biplot analyses require is a two-way data matrix, such as number of genotypes tested in a number of environments. The experiment may or may not be replicated. These analyses combine two statistical procedures: analysis of variance (ANOVA) and principal component analysis (PCA) (Gauch, 2006). The purpose of this research was to apply GGE biplot and AMMI techniques to study the patterns of $\mathrm{G} \times \mathrm{E}$ interaction in maize; to graphically display means, adaptability and stability of maize inbred lines and to identify suitable inbred lines for each sowing date.

\section{MATERIALS AND METHODS}

Sixteen maize inbred lines Table 1 were evaluated in four sowing date during two seasons of 2012 and 2013 using a randomized complete block design (RCBD) with four replications at Sakha Research Station, Kafr, ElSheikh Governorate. The four sowing date were $1^{\text {st }}$ May (D1), $15^{\text {th }}$ May (D2), $1^{\text {st }}$ Jun (D3), and $15^{\text {th }}$ Jun (D4). The same 16 inbred lines were used in each sowing date. All agronomic practices were carried out as recommended. Each plot consisted of two rows, $80 \mathrm{~cm}$ in width, $6 \mathrm{~m}$ in length and $20 \mathrm{~cm}$ between hills, all plants are harvested for grain yield and adjusted to $15.5 \%$ moisture, and Bartlet's test of homogeneity of variances and least significant difference test at $5 \%$ level of probability was used to compare means. The ANOVA and Graphic analysis (GGE biplot) was performed using GGE biplot software (Yan, 2001). AMMI and GGE biplot methods were used to study the $G, E$ and $G \times E$ effects on grain yield. These methods have been described in detail by Gabriel (1971), Yan et al. (2001), Yan (2002), Yan and Hunt (2002), Yan and Kang (2003) and Gauch (1992 and 2006).

Table 1: The codes and names of maize inbred lines

\begin{tabular}{|l|c|c|c|}
\hline Inbred lines codes & Inbred lines names & Inbred lines codes & Inbred lines names \\
\hline Gm 2 & Gemmeiza 2 & $\mathrm{Gz} \mathrm{602}$ & Giza 602 \\
\hline $\mathrm{Gm} 4$ & Gemmeiza 4 & $\mathrm{Gz} \mathrm{603}$ & Giza 603 \\
\hline Sd 7 & Sids7 & $\mathrm{Gz} \mathrm{612}$ & Giza 612 \\
\hline $\mathrm{Gm} \mathrm{18}$ & Gemmeiza 18 & $\mathrm{Gz} \mathrm{628}$ & Giza 628 \\
\hline $\mathrm{Gm} 21$ & Gemmeiza 21 & $\mathrm{GZ} \mathrm{629}$ & Giza 629 \\
\hline $\mathrm{Gm} 27$ & Gemmeiza 27 & $\mathrm{Gm} \mathrm{1002}$ & Gemmeiza 1002 \\
\hline Sd 34 & Sids 34 & $\mathrm{Gm} \mathrm{1004}$ & Gemmeiza 1004 \\
\hline Sd 63 & Sids 63 & $\mathrm{Gm} \mathrm{1021}$ & Gemmeiza 1021 \\
\hline
\end{tabular}




\section{RESULTS AND DISCUSSION}

Regarding to interaction effects data in Table 2 showed that interaction between sowing dates and maize inbred had highly significant effects on all studied traits in both seasons. Gz 603, Sd 34 and Gz 629 inbred had highest grain yield in both seasons under the $2^{\text {nd }}$ sowing date. Whereas, these inbred recorded $26.09,21.01$, and 20.09 ardab/fed, respectively in the $1^{\text {st }}$ season and 28.59, 23.51 and $20.79 \mathrm{ardab} / \mathrm{fed}$, respectively in the $2^{\text {nd }}$ season under the second sowing date for yield.

Table 2:Comparative yield performance of maize (Zea mays L.) inbred lines at different sowing dates during 2012 and 2013 seasons.

\begin{tabular}{|c|c|c|c|c|c|c|c|c|c|c|c|c|}
\hline \multirow{3}{*}{$\begin{array}{l}\text { Inbred } \\
\text { lines }\end{array}$} & \multicolumn{12}{|c|}{ sowing dates 2012} \\
\hline & \multicolumn{4}{|c|}{$\begin{array}{c}\text { No of days to } 50 \% \\
\text { tasseling }\end{array}$} & \multicolumn{4}{|c|}{$\begin{array}{c}\text { No of days to } 50 \% \\
\text { silking }\end{array}$} & \multicolumn{4}{|c|}{$\begin{array}{l}\text { Plant } \\
\text { height }\end{array}$} \\
\hline & $\begin{array}{c}1^{\text {st }} \\
\text { MAY }\end{array}$ & $\begin{array}{c}15^{\text {th }} \\
\text { MAY }\end{array}$ & $\begin{array}{c}1^{\mathrm{st}} \\
\mathrm{JUN}\end{array}$ & $\begin{array}{l}15^{\mathrm{th}} \\
\text { JUN }\end{array}$ & $\begin{array}{c}1^{\text {st }} \\
\text { MAY }\end{array}$ & $\begin{array}{l}15^{\mathrm{th}} \\
\text { MAY }\end{array}$ & $\begin{array}{c}1^{\text {st }} \\
\text { JUN }\end{array}$ & $\begin{array}{l}15^{\mathrm{th}} \\
\text { JUN }\end{array}$ & $\begin{array}{c}1^{\text {st }} \\
\text { MAY }\end{array}$ & $\begin{array}{l}15^{\text {th }} \\
\text { MAY }\end{array}$ & $\begin{array}{c}1^{\mathrm{st}} \\
\mathrm{JUN}\end{array}$ & $\begin{array}{l}15^{\mathrm{th}} \\
\text { JUN }\end{array}$ \\
\hline GM 2 & 80.25 & 74.00 & 72.50 & 69.25 & 79.00 & 73.50 & 73.00 & 69.25 & 199.75 & 133.75 & 111.50 & 96.00 \\
\hline GM 4 & 72.25 & 66.00 & 68.00 & 67.25 & 73.25 & 67.00 & 69.00 & 68.00 & 214.75 & 214.25 & 164.750 & 1.00 \\
\hline Sd 7 & 72.25 & 69.00 & 70.50 & 70.25 & 72.00 & 70.75 & 71.50 & 70.50 & 257.75 & 255.00 & 178.25 & 191.50 \\
\hline GM 18 & 76.00 & 70.50 & 72.00 & 69.50 & 77.25 & 71.75 & 73.75 & 70.25 & 185.75 & 185.00 & 132.75 & 124.25 \\
\hline GM 21 & 74.00 & 66.00 & 66.75 & 68.25 & 74.25 & 67.50 & 68.25 & 69.25 & 201.00 & 206.00 & 163.50 & 155.00 \\
\hline GM 27 & 76.00 & 69.50 & 74.50 & 72.25 & 77.25 & 71.00 & 76.50 & 72.75 & 216.50 & 217.75 & 172.50 & 170.00 \\
\hline Sd 34 & 71.50 & 65.75 & 68.25 & 67.00 & 72.25 & 67.00 & 69.25 & 67.75 & 231.50 & 236.50 & 188.50 & 192.75 \\
\hline Sd 63 & 76.25 & 69.75 & 72.50 & 71.00 & 75.50 & 71.75 & 74.25 & 71.50 & 205.25 & 193.75 & 177.00 & 139.00 \\
\hline Gz 602 & 72.75 & 68.00 & 69.25 & 69.25 & 73.25 & 69.00 & 69.50 & 69.75 & 253.00 & 279.00 & 195.00 & 210.00 \\
\hline Gz 603 & 72.25 & 66.50 & 70.50 & 69.00 & 72.50 & 66.75 & 71.50 & 69.25 & 261.25 & 255.75 & 190.25 & 222.75 \\
\hline Gz 612 & 71.50 & 67.75 & 70.00 & 65.00 & 71.75 & 66.25 & 71.25 & 66.25 & 264.00 & 270.75 & 210.75 & 224.00 \\
\hline Gz 628 & 72.50 & 66.50 & 68.25 & 68.25 & 72.25 & 66.50 & 69.50 & 68.00 & 211.25 & 204.75 & 152.25 & 153.25 \\
\hline Gz 629 & 70.50 & 65.00 & 66.00 & 65.25 & 70.75 & 65.50 & 67.75 & 67.50 & 199.75 & 209.25 & 152.75 & 134.00 \\
\hline $\mathrm{Gm} 1002$ & 69.25 & 63.00 & 64.25 & 65.25 & 69.25 & 63.75 & 66.75 & 66.25 & 209.00 & 216.50 & 163.25 & 164.25 \\
\hline Gm 1004 & 72.25 & 67.25 & 69.00 & 69.00 & 72.00 & 68.5 & 69.25 & 69.25 & 208.25 & 201.25 & 163.25 & 150.25 \\
\hline $\mathrm{Gm} 1021$ & 71.75 & 65.25 & 65.75 & 64.00 & 71.25 & 65.25 & 65.50 & 64.00 & 238.25 & 228.50 & 167.50 & 164.75 \\
\hline L. S. D & \multicolumn{4}{|c|}{3.02} & \multicolumn{4}{|c|}{3.1} & \multicolumn{4}{|c|}{22.58} \\
\hline
\end{tabular}

\section{Continue}

\begin{tabular}{|c|c|c|c|c|c|c|c|c|c|c|c|c|}
\hline \multirow[t]{2}{*}{ Inbred } & \multicolumn{4}{|c|}{ Ear height } & \multicolumn{4}{|c|}{ Ear } & \multicolumn{4}{|c|}{ Yield } \\
\hline & $\begin{array}{c}1^{\mathrm{St}} \\
\text { MAY }\end{array}$ & $\begin{array}{l}15^{\text {th }} \\
\text { MAY }\end{array}$ & $\begin{array}{c}1^{\text {st }} \\
\text { JUN }\end{array}$ & $\begin{array}{l}15^{\mathrm{th}} \\
\text { JUN }\end{array}$ & $\begin{array}{c}1^{\text {st }} \\
\text { MAY }\end{array}$ & $\begin{array}{l}15^{\text {th }} \\
\text { MAY }\end{array}$ & $\begin{array}{c}1^{\text {st }} \\
\text { JUN }\end{array}$ & $\begin{array}{l}15^{\text {th }} \\
\text { JUN }\end{array}$ & $\begin{array}{c}1^{\text {St }} \\
\text { MAY }\end{array}$ & $\begin{array}{l}15^{\text {th }} \\
\text { MAY }\end{array}$ & $\begin{array}{c}1^{\text {st }} \\
\text { JUN }\end{array}$ & $\begin{array}{l}15^{\text {th }} \\
\text { JUN }\end{array}$ \\
\hline GM 2 & 105.25 & 99.75 & 67.25 & 61.00 & 8.00 & 7.00 & 2.75 & 3.75 & 1.98 & 2.16 & 0.835 & 1.17 \\
\hline GM 4 & 11.75 & 114.75 & 92.75 & 65.75 & 34.50 & 37.25 & 34.75 & 28.00 & 8.66 & 10.46 & 8.27 & 6.01 \\
\hline Sd 7 & 7.00 & 139.50 & 108.00 & 96.50 & 59.25 & 54.25 & 36.50 & 29.00 & 17.46 & 15.98 & 10.4 & 7.88 \\
\hline GM 18 & 5.25 & 116.75 & 85.75 & 73.50 & 42.75 & 51.75 & 24.25 & 30.25 & 6.88 & 10.56 & 3.96 & 5.72 \\
\hline GM & 7.75 & 111.75 & 99.00 & 80.75 & 35.75 & 47.75 & 43.25 & 38.25 & 44 & 11.71 & 7.62 & 6.68 \\
\hline$G$ & 9.25 & 125.50 & 105.75 & 04.25 & 5.00 & 47.25 & 47.50 & 39.00 & 8.26 & 13.22 & 7.62 & 5.33 \\
\hline $\mathrm{Sd}$ & 2.75 & 132.00 & 04.25 & 94.75 & 49.50 & 55.5 & 50.75 & 44.25 & 18.93 & 21.01 & 16.80 & 12.91 \\
\hline $\mathrm{Sd}$ & 2.00 & 124.75 & 100.50 & 98.00 & 41.75 & 48.25 & 38.25 & 26.50 & 11.47 & 13.12 & 9.49 & 3.86 \\
\hline $\mathrm{Gz} 602$ & 140.00 & 149.25 & 103.75 & 98.25 & 52.75 & 51.00 & 51.50 & 48.50 & 9.61 & 14.2 & 12.51 & 15.11 \\
\hline $\mathrm{Gz} 603$ & 147.50 & 143.75 & 116.25 & 117.50 & 63.25 & 79.25 & 47.00 & 49.25 & 18.16 & 26.09 & 13.81 & 13.77 \\
\hline $\mathrm{Gz} 612$ & 41.00 & 140.00 & 109.50 & 105.75 & 60.25 & 59.00 & 49.50 & 43.25 & 13.68 & 15.75 & 11.96 & 10.6 \\
\hline Gz 628 & 124.75 & 118.75 & 95.75 & 91.50 & 46.25 & 63.25 & 48.50 & 40.75 & 10.98 & 19.73 & 12.34 & 8.44 \\
\hline $\mathrm{Gz}$ & 4.75 & 112.25 & 92.75 & 71.75 & 46.00 & 54.00 & 50.25 & 33.00 & 17.55 & 20.09 & 12.45 & 7.84 \\
\hline $\mathrm{Gm}$ & 14.00 & 114.75 & 98.75 & 81.00 & 34.50 & 29.75 & 34.25 & 37.75 & 6.11 & 7.21 & 5.75 & 6.88 \\
\hline $\mathrm{Gm}$ & 122.75 & 112.75 & 98.50 & 79.75 & 48.00 & 48.5 & 39.75 & 28.75 & 15.29 & 16.21 & 12.17 & 8.24 \\
\hline $\mathrm{Gm} 1021$ & 31.25 & 119.25 & 96.00 & 84.25 & 43.50 & 44.5 & 43.25 & 38.25 & 13.98 & 13.69 & 10.07 & 6.68 \\
\hline S. D & \multicolumn{4}{|c|}{16.46} & \multicolumn{4}{|c|}{11.94} & \multicolumn{4}{|c|}{3.1} \\
\hline
\end{tabular}




\section{Continue}

\begin{tabular}{|c|c|c|c|c|c|c|c|c|c|c|c|c|}
\hline \multirow[t]{3}{*}{ Inbred } & \multicolumn{12}{|c|}{ sowing dates 2013} \\
\hline & \multicolumn{4}{|c|}{$\begin{array}{c}\text { No of days to } 50 \% \\
\text { tasseling }\end{array}$} & \multicolumn{4}{|c|}{$\begin{array}{c}\text { No of days to } 50 \% \\
\text { silking }\end{array}$} & \multicolumn{4}{|c|}{$\begin{array}{l}\text { Plant } \\
\text { height }\end{array}$} \\
\hline & \begin{tabular}{|c|}
$1^{\text {st }}$ \\
MAY
\end{tabular} & \begin{tabular}{|c|}
$15^{\text {th }}$ \\
MAY
\end{tabular} & $\begin{array}{c}1^{\mathrm{st}} \\
\text { JUN }\end{array}$ & \begin{tabular}{|l|}
$15^{\text {th }}$ \\
JUN
\end{tabular} & $\begin{array}{c}1^{\text {st }} \\
\text { MAY }\end{array}$ & \begin{tabular}{|c|}
$15^{\text {th }}$ \\
MAY
\end{tabular} & $\begin{array}{c}1^{\text {st }} \\
\text { JUN }\end{array}$ & \begin{tabular}{|c|}
$15^{\text {th }}$ \\
JUN
\end{tabular} & $\begin{array}{c}1^{\text {st }} \\
\text { MAY }\end{array}$ & $\begin{array}{l}15^{\text {th }} \\
\text { MAY }\end{array}$ & $\begin{array}{c}1^{\text {st }} \\
\text { JUN }\end{array}$ & $\begin{array}{l}15^{\text {th }} \\
\text { JUN }\end{array}$ \\
\hline 12 & 75.250 & 3.750 & 68.000 & 64.750 & 75.500 & 79.500 & 67.750 & 65.750 & 152.750 & 166.500 & 188.25 & 136.250 \\
\hline GM 4 & 71.500 & 500 & 61.250 & 61.250 & 71.750 & 72.250 & 61.750 & 63.750 & 197.250 & 500 & 190.000 & 2.500 \\
\hline Sd 7 & 68.500 & 2.750 & 63.250 & 64.000 & 71.750 & 73.000 & 62.750 & 64.750 & 233.25 & 240.500 & 228.750 & 217.000 \\
\hline GM 18 & 73.500 & 5.750 & 67.000 & 65.750 & 74.250 & 78.750 & 67.500 & 65.500 & 184.250 & 193.750 & 178.250 & 159.750 \\
\hline GM 21 & 71.000 & 0.500 & 61.500 & 63.50 & 71.500 & 73.750 & 63.250 & 64.250 & 188.250 & 189.750 & 222.500 & 181.250 \\
\hline GM 27 & 73.25 & 75.250 & 67.000 & 66.500 & 73.750 & 78.000 & 67.250 & 67.000 & 235.500 & 221.750 & 208.500 & 209.250 \\
\hline Sd 34 & 71.000 & 72.000 & 62.750 & 62.750 & 71.75 & 73.000 & 62.750 & 64.500 & 232.250 & 238.750 & 232.500 & 221.750 \\
\hline Sd 63 & 0 & 76.50 & 67.500 & 63.750 & 73.500 & 00 & 66.00 & 65.250 & 188.250 & & 193.500 & 168.000 \\
\hline Gz 602 & 73.250 & 74.750 & 65.000 & 65.250 & 73.750 & 74.750 & 65.500 & 65.500 & 239.500 & 250 & 263.250 & 247.750 \\
\hline Gz 603 & 70.500 & 72.500 & 63.250 & 64.000 & 70.500 & 72.500 & 63.500 & 65.250 & 259.000 & 25 & 241.500 & 213.250 \\
\hline Gz 612 & 70.250 & 71.750 & 62.500 & 63.500 & 70.500 & 72.250 & 62.500 & 65.750 & 259.250 & 251.750 & 241.000 & 226.000 \\
\hline Gz 628 & 71.250 & 72.50 & 63.500 & 63.000 & 71.750 & 73.000 & 62.500 & 63.000 & 190.000 & 212.00 & 194.500 & \begin{tabular}{|l|}
181.250 \\
\end{tabular} \\
\hline Gz 629 & 70.500 & 71.000 & 60.750 & 59.50 & 71.000 & 71.250 & 60.750 & 60.250 & 200.750 & 208.250 & 211.250 & 176.750 \\
\hline $\begin{array}{l}\mathrm{Gm} \\
1002 \\
\end{array}$ & 00 & 50 & 750 & .500 & .500 & 000 & 59.750 & 250 & 38.000 & 00 & 225.000 & 209.000 \\
\hline $\begin{array}{l}\mathrm{Gm} \\
1004\end{array}$ & 71.750 & 72.250 & 62.750 & 61.250 & 73.000 & 74.500 & 62.000 & 61.500 & 213.750 & 219.500 & 195.000 & 183.750 \\
\hline $\begin{array}{l}\mathrm{Gm} \\
1021\end{array}$ & 70.250 & 70.500 & 60.750 & 60.000 & 71.000 & 70.750 & 60.250 & 60.250 & 214.000 & 228.250 & 226.000 & 197.500 \\
\hline L.S. D & 2.16 & & & & 1.8 & & & & 26.57 & & & \\
\hline
\end{tabular}

\section{Continue}

\begin{tabular}{|c|c|c|c|c|c|c|c|c|c|c|c|c|}
\hline \multirow[t]{2}{*}{ Inbred } & \multicolumn{4}{|c|}{ Ear height } & \multicolumn{4}{|c|}{ Ear } & \multicolumn{4}{|c|}{ Yield } \\
\hline & $\begin{array}{l}1^{\text {st }} \\
\text { MAY }\end{array}$ & $\begin{array}{l}15^{\mathrm{ln}} \\
\text { MAY }\end{array}$ & $\begin{array}{c}1^{\text {st }} \\
\text { JUN }\end{array}$ & $\begin{array}{l}15^{\mathrm{th}} \\
\text { JUN }\end{array}$ & $\begin{array}{c}1^{\text {st }} \\
\text { MAY }\end{array}$ & $\begin{array}{l}15^{\mathrm{th}} \\
\text { MAY }\end{array}$ & $\begin{array}{c}1^{\text {st }} \\
\text { JUN }\end{array}$ & \begin{tabular}{|l|}
$15^{\mathrm{th}}$ \\
JUN
\end{tabular} & \begin{tabular}{|c|}
$1^{\text {st }}$ \\
MAY
\end{tabular} & \begin{tabular}{|l|}
$15^{\mathrm{ln}}$ \\
MAY
\end{tabular} & $\begin{array}{c}1^{\mathrm{st}} \\
\text { JUN }\end{array}$ & $\begin{array}{l}15^{\mathrm{th}} \\
\text { JUN }\end{array}$ \\
\hline 12 & 105.25 & 9.75 & 67.25 & .00 & 97.750 & 93.25 & 113.5 & 85.0 & 3.43 & 5.58 & 4.28 & 5.77 \\
\hline GM 4 & 5 & 4.75 & 92.75 & 75 & 5.25 & 94.25 & .50 & 00.5 & .89 & 7.22 & 12.9 & 5.24 \\
\hline Sd 7 & 147.00 & 9.50 & 108.00 & 96.50 & 31.25 & 115.5 & 122.8 & 111.8 & 19.34 & 12.72 & 17.66 & 9.15 \\
\hline GM 18 & 5.25 & 6.75 & 85.75 & 73.50 & 111.0 & 101.5 & 107.0 & 101.0 & \begin{tabular}{|l|l|}
8.41 \\
\end{tabular} & 7.97 & 8.51 & 5.79 \\
\hline GM 21 & 107.75 & 11.75 & 99.00 & 80.75 & 104.25 & 91.75 & 127.5 & 103.8 & 8.95 & 8.99 & 11.35 & 5.74 \\
\hline M 27 & 29.25 & 25.50 & 105.75 & 104.25 & 136.0 & 116.8 & 120.8 & 116.8 & 11.23 & 9.49 & 1.44 & 3.68 \\
\hline Sd 34 & 132.75 & 0 & 104.25 & 94.75 & 134.50 & 121.3 & 136.5 & 132.3 & 24.23 & 23.51 & 21.73 & 12.64 \\
\hline 63 & 122.00 & 5 & 100.50 & 98 & 107.5 & 110.3 & 117.3 & 5.5 & 89 & 13.88 & 11.09 & 5.04 \\
\hline $\mathrm{Gz} 602$ & $\overline{0}$ & 5 & 103. & 3.25 & 133.50 & 124.0 & 143.3 & 134.3 & 16.21 & 10.16 & 17.68 & 10.06 \\
\hline Gz 603 & 147.50 & 3.75 & 116.25 & 117.50 & 150.5 & 144.3 & 147.5 & 129.5 & 17.41 & 28.59 & 23.69 & 14.32 \\
\hline Gz 612 & 141.00 & 140.00 & 109.50 & 105.75 & 134.75 & 128.5 & 129.8 & 107.3 & 21.84 & 11.48 & 5.92 & 5.03 \\
\hline Gz 628 & 124.75 & 8.75 & 95.75 & 91.50 & 16.25 & 117.0 & 113.3 & 111.3 & 9.27 & 6.58 & 5.71 & 11.5 \\
\hline Gz 629 & 114.75 & 112.25 & 92.75 & 71.75 & 108.25 & 111.3 & 123.8 & 98.75 & 18.26 & 20.79 & 16.59 & 12.03 \\
\hline $\mathrm{Gm}$ & & 5 & 75 & 0 & 25 & 88.75 & 125.3 & 116.3 & 7.44 & 9.68 & 10.49 & 5.02 \\
\hline & 2.75 & 112.75 & 98.50 & .75 & 2.50 & 1.5 & 116.3 & 101.3 & 15.56 & 9.98 & 15.44 & 10.95 \\
\hline & & 9.25 & 96.00 & 84.25 & 110 & 115.3 & 131 & 112.3 & $<0.05$ & 7.23 & 16. & 6.92 \\
\hline$\pi$ & & & & & & & & & & & & \\
\hline
\end{tabular}

The ANOVA for grain yield using the AMMI method is presented in Tables 3, 4 and 5 for 2012 and 2013 seasons and the average of the two seasons, respectively. There were significant differences among the inbred lines $(G)$, sowing dates $(D)$ and $G \times D$ interaction. Significant $G \times D$ interaction explain 1 $1.45 \%, 12.69 \%$ and $9.01 \%$ of the total sum of squares for 2012 and 2013 and the two-year average, respectively. 
Table 3: Analysis of variance for grain yield of 16 maize inbred lines in four sowing dates during the 2012 cropping season

\begin{tabular}{|l|c|c|c|c|c|}
\hline S.O.V & Df & SS & MS & P $>$ F & $\%$ of Total \\
\hline Inbred lines (G) & 15 & 4320 & $288.0^{*}$ & $<0.00000$ & 54.41 \\
\hline Planting dates (D) & 3 & 1485 & $494.9^{*}$ & $<0.00000$ & 18.71 \\
\hline DXG & 45 & 909 & $20.2^{*}$ & $<0.00000$ & 11.45 \\
\hline IPC1 & 17 & 589 & $34.6^{*}$ & $<0.00000$ & 7.42 \\
\hline IPC2 & 15 & 242 & 16.1 & $<0.00002$ & 3.05 \\
\hline Residual & 13 & 78 & 6.0 & & \\
\hline Error & 180 & 813 & 4.5 & & 10.24 \\
\hline Total & 255 & 7939 & 31.1 & & \\
\hline
\end{tabular}

Table 4: Analysis of variance for grain yield of 16 maize inbred lines in four sowing dates during the 2013 cropping season

\begin{tabular}{|l|c|c|c|c|c|}
\hline S.O.V & Df & SS & MS & $\mathbf{P}>\mathbf{F}$ & $\%$ of Total \\
\hline Inbred lines (G) & 15 & 4702 & $313.5^{*}$ & $<0.00000$ & 50.05 \\
\hline Planting dates (D) & 3 & 2532 & $844.0^{*}$ & $<0.00000$ & 26.95 \\
\hline DXG & 45 & 1192 & $26.5^{*}$ & $<0.00000$ & 12.69 \\
\hline IPC1 & 17 & 621 & 36.5 & $<0.00000$ & 6.61 \\
\hline IPC2 & 15 & 315 & 21.0 & $<0.00000$ & 3.35 \\
\hline Residual & 13 & 257 & 19.8 & & \\
\hline Error & 180 & 913 & 5.1 & & 9.72 \\
\hline Total & 255 & 9395 & 36.8 & & \\
\hline
\end{tabular}

Table 5: Analysis of variance for grain yield of 16 maize inbred lines in four planting dates during the 2012 and 2013 cropping seasons

\begin{tabular}{|l|c|c|c|c|c|}
\hline S.O.V & Df & SS & MS & P $>$ F & $\%$ of Total \\
\hline Inbred lines (G) & 15 & 4344 & $289.6^{\text {* }}$ & $<0.00000$ & 59.13 \\
\hline Planting dates (D) & 3 & 1800 & $599.8^{* \prime}$ & $<0.00000$ & 24.49 \\
\hline DXG & 45 & 662 & $14.7^{\prime \prime}$ & $<0.00000$ & 9.01 \\
\hline IPC1 & 17 & 423 & $24.9^{*}$ & $<0.00000$ & 5.76 \\
\hline IPC2 & 15 & 157 & $10.5^{*}$ & $<0.00000$ & 2.14 \\
\hline Residual & 13 & 82 & 6.3 & & \\
\hline Error & 180 & 427 & 2.4 & & 5.81 \\
\hline Total & 255 & 7347 & 28.8 & & \\
\hline
\end{tabular}

The AMMI analysis also identified the best planting date as D2 which had the highest PCA1 and the best 1PCA2 values of 1.84 and 1.59, respectively (Table 6). However, high PCAs show unstable yields which could be used in the selection site for genotypes to be grown in specific environments (Akcura et al., 2011). Thus, this study identified the sowing dates which optimized genotype selection on the basis of their discriminating ability and representativeness. From the AMMI analysis, inbred lines (G10) Giza 603 and (G7) Sids34 performed well in two of these sowing dates (Table $6)$. 
Table 6: Sowing dates ranked on IPCA scores including the first four recommended inbred lines for each sowing dates based on AMMI the estimates

\begin{tabular}{|l|c|c|c|c|c|c|c|}
\hline $\begin{array}{l}\text { Sowing } \\
\text { dates }\end{array}$ & Dm & IPCAe[1] & IPCAe[2] & $\mathbf{1}$ & $\mathbf{2}$ & $\mathbf{3}$ & $\mathbf{4}$ \\
\hline D1 & 12.02 & 1.00166 & -1.84476 & Giza603 & Sids34 & Giza629 & Giza628 \\
\hline D2 & 15.48 & 1.83742 & 1.59545 & Sids34 & Giza603 & Giza629 & Gemmeiza1021 \\
\hline D3 & 12.16 & -0.45164 & 0.25233 & Sids34 & Giza603 & Giza629 & Giza628 \\
\hline D4 & 8.00 & -2.38744 & -0.50165 & Giza603 & Sids34 & Giza602 & Giza628 \\
\hline
\end{tabular}

The first two PCs explained $94.80 \%, 91.18 \%$ and $96.31 \%$ of the total GGE variation in data for 2012 and 2013 sesons and the two-year average, respectively. The graphical method was employed to investigate sowing dates variation and interpret the $G \times D$ interaction Fig. 1.The ranking of 16 inbred lines based on their mean grain yield and yield stability for 2012 and 2013 seasons and the two-year average, respectively, is shown in Fig. 1. It has been reported that when PC1 in a GGE biplot approximates the lines (mean performance), PC2 must approximate the $G \times D$ associated with each genotype, which is a measure of instability (Yan et al., 2000; Yan, 2002). The line passing through the biplot origin and the sowing dates average is indicated by circles and is known as the average sowing dates coordinate (ADC) axis, which is defined by the average PC1 and PC2 scores for all sowing dates. Projection of genotype markers on to this axis should, therefore, approximate the mean yield of the genotypes. Thus, inbred lines Sids 34, Giza 629, Gemmeiza 1021, Sids7, Giza 612, Giza628, Gz603, Gemmeiza 1004 and Giza602 had higher grain yield, followed by genotypes Gemmeiza 2, Gemmeiza 18, Gemmeiza 27, Sids 63, Gemmeiza 2, Gemmeiza 4 and Gemmeiza 1002 for all data set. The line which passes through the origin but is perpendicular to the ADC with double arrows represents the status of the genotypes' stability. A position in either direction away from the biplot origin, on this axis, indicates greater $G \times D$ interaction and reduced stability (Yan, 2002). Therefore, inbred lines Gemmeiza 1021, Sids63 and Giza602 showed a more variable and less stable performance than the other genotypes. Genotypes Giza603, Sids 34, Giza 628, Gemmeiza 2, Gemmeiza 4, Gemmeiza 1002 and Gemmeiza 18 in the two-year average were more stable than the others Fig. 1.

Fig. 2 provides a summary of the interrelationships among sowing dates. The lines connecting the biplot origin and the markers for the sowing dates are called sowing dates vectors. The angle between the vectors of two sowing dates is related to the correlation coefficient between them. Based on the angles of the sowing dates vectors, the four sowing date were clustered into two groups in all cases. Group one included first sowing date (D1) and second sowing date (D2) Group two included third sowing date (D3) and fourth sowing date (D4). 


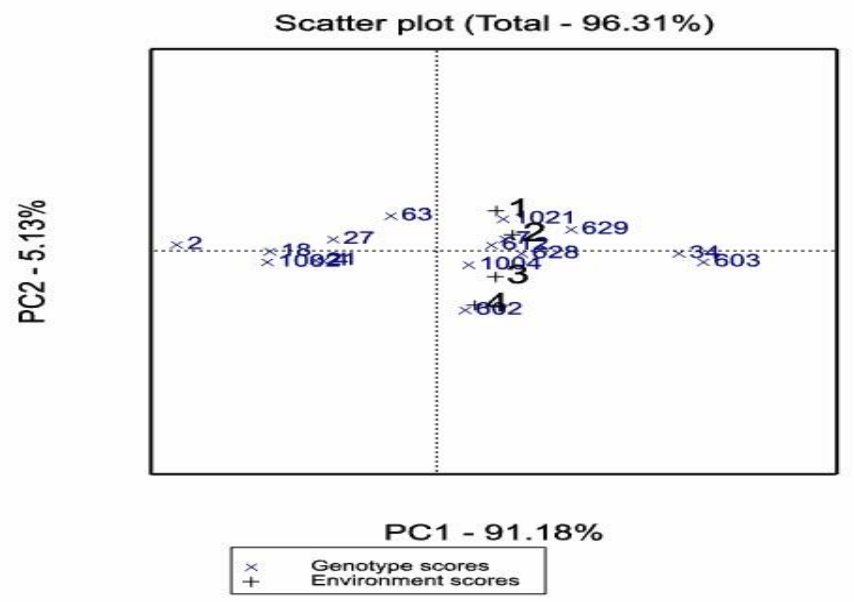

Figure 1: The Average Tester Coordination View (Genotype focus scaling)

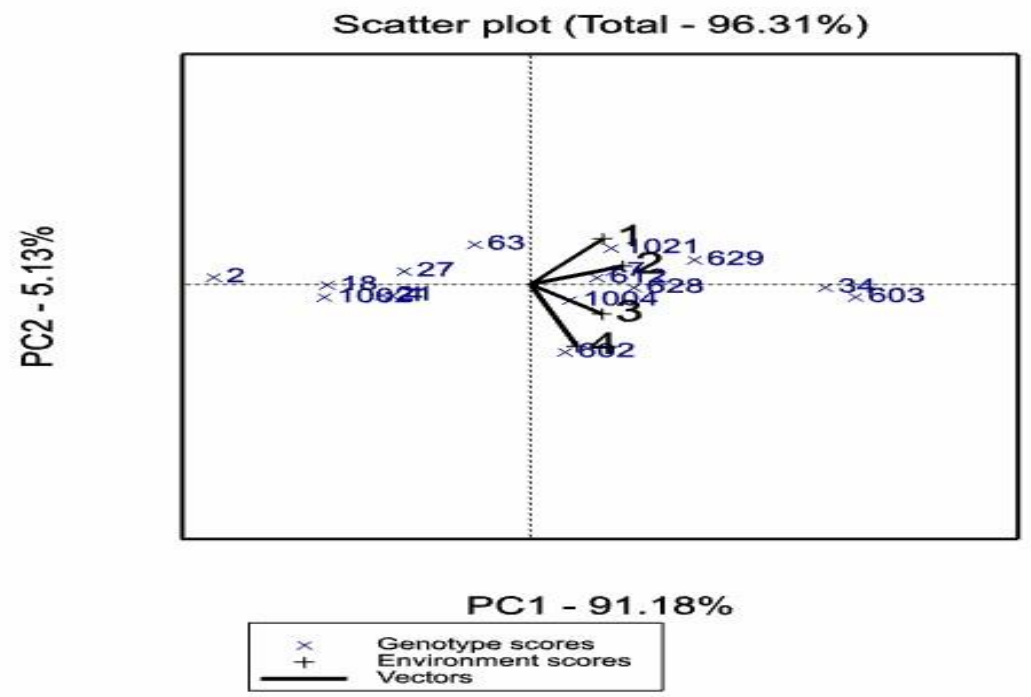

Figure 2: Discriminating power and representativeness of test environments 
For example, the fact that the smallest angle is between first, second sowing date and third, fourth sowing date implies that there was the highest correlation between them. The large angle between D1 and D4indicates the poor correlation between these locations Fig. 2. Another interesting observation from the vector point of view of the biplot is that the length of the environment vectors approximates the standard deviation within each environment, which is a measure of its discriminating ability (Yan and Kang, 2003). Thus sowing date 1 and 4 are the most discriminative sowing dates Fig. 2. Another important feature of a test sowing date is how much it represents the target sowing date. To measure representativeness using a biplot, an average sowing date has to be defined and used as a reference. Inbred lines Sids34, Giza 629, Gemmeiza 1021, Sids 7, Giza 612, Giza 628, Giza 603, Gemmeiza 1004 and Giza 602 had higher grain yield than the grand mean in sowing date1. Sowing dates could also be ranked according to one inbred line. Inbred line Giza 603 had the maximum grain yield and high yield stability Fig 1.

Sowing dates are ranked along this axis in the direction of the dot representing inbred line Giza 603 in Fig. 2. For example, the relative performance of line Giza 603 in different sowing dates in Fig. 2 ranks as follows: D2>D3>D1>D4. The line perpendicular to the inbred line Giza603 axis separates sowing dates in which inbred line Giza 603is below and above the mean. However, inbred line Giza603 is above the mean in all four sowing dates. It could be summarized that the 16 maize inbred lines showed very high variation for grain yield.- The four sowing date were clustered into two groups in all cases.- Inbred line Giza 603 the best performer in all inbred lines, followed by Sids 34, Giza 629 and Giza 628 - Inbred lines Giza 603, Sids 34 , Giza 628 , had the highest yield stability into the best sowing dates. Thus sowing date 1 and 4 are the most discriminative sowing dates. This inbred lines intervention in the production of many commercial hybrids.

\section{REFERENCES}

A I. H., K. E . Basford, M. Cooper ; J. K. Bull, and C. G.McLaren (1996). Analysis of multi-environment trials a historical perspective. Pp. 39-124. In M.Cooper and G. L. Hammer (eds.). Plant adaptation and crop improvement. CAB International Iran.

Gabriel, K. R. (1971). The biplot graphic of matrices with application to principal component analysis. Biometrics, 58: 453-467.

Gauch, H. G. (1992). Statistical analysis of regional yield trials: AMMI analysis of factorial designs. Elsevier, Amsterdam, The Netherlands. PP 278.

Gauch, H. G. (2006). Statistical analysis of yield trials by AMMI and GGE. Crop Sci. 46: 1488-1500.

Hammer, G. L. and M. Cooper (1996). Plant Adaptation and Crop Improvement. CAB International. 608pp. 
Kaya, Y., M. Akcura and S. Taner( 2006). GGE- biplot analysis of multienvironment yield trials in bread wheat. Turk. J. Agric., 30: 325337.

Lee, S., W. Yan and J. K. Ahn( 2003). Effects of year, site, genotype their interactions on various soybean isoflavones. Field Crops Res. 81: 181-192.

Pacheco and, R. M. and D. J. B. Vencovsky (2005).Use of supplementary genotypes in AMMI analysis. Theor.Appl. Genet.,110: 812-818.

Samonte, S.O.P.B.; L. T. Wilson; A. M. McClung and J.C. Medley (2005). Targeting cultivars onto ricegrowing environments using AMMI and SREG GGE biplotanalysis. Crop Sci., 45(6): 2414-2424.

$\mathrm{Wu}, \mathrm{H}$. X. and A. C. Matheson (2005) . Genotype by environment interactions in an Australia-wide radiate pine diallel mating experiment: implications for regionalized breeding. Forest Sci., 51(1): 29-40.

Yan, W. (2001). GGE biplot - A Windows application for graphical analysis Of multi-environment trial data and other types of two-way data. Agron. J., 93: 1111-1118.

Yan, W.( 2002). Singular-value partitioning in biplot analysis of multienvironment trial data. Agron. J., 94:990-996.

Yan, W. P. L. Cornelius, J. Crossa, and L. A. Hunt (2001).Two typesof GGE biplots for analysis of multi environment trial data. Crop Sci., 41: 565-663.

Yan, W. and L. A. Hunt (2002). Biplot analysis of multienvironment trial data. Pp. 289-303. In M. S. Kang (ed.).Quantitative Genetics, Genomics and Plant Breeding. CAB International Iran.

Yan, W. and M. S. Kang( 2003). GGE biplot analysis: agraphical tool for breeders, geneticists andagronomist. CRC Press, Boca Raton, FL. PP. 271

Yan, W. L. A. Hunt; Q. Sheng and Z. Szlavnics (2000). Cultivar evaluation and mega-environment investigation based on the GGE biplot. Crop Sci. 40:597-605.

Yan, W. and N. A. Tinker (2005). An integrated system of biplot analysis for displaying, interpreting, and exploring genotype by environment interactions. Crop Sci., 45:1004-1016. 
استخدام طرق احصـائية لتقييم بعض سـلالات الذرة الثـامية تحت مواعيد زراعة مختلفة

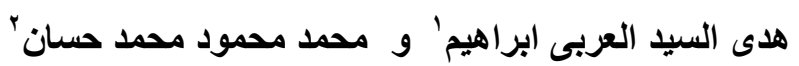

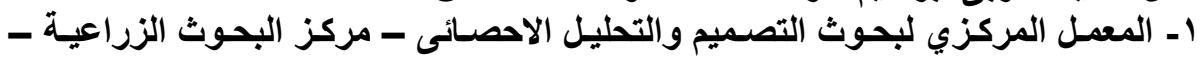

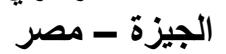
r - معهد بحوث المحاصيل الحقلية ـ مركز البحوث الزراعية ـ الجيزة ـ مصر

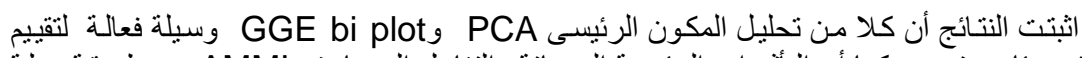

المحصول فى بيئات متعددة .كما أن النأثيرات الرئيسية المضافة والثفاعل المضاعف AMMI هى طريقة بديلة

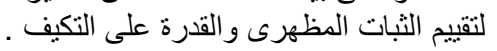

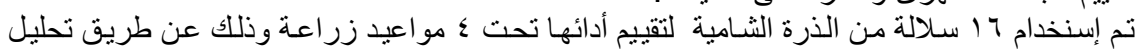
AMMI, GGE bi plot

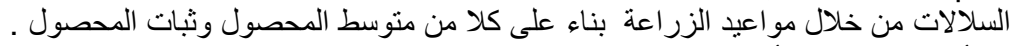

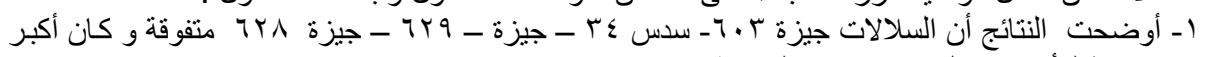
متوسط للأداء بين السلالات تحت التحت الدراسة. r- تم إتتخدام تحليل الرسوم البيانية لتحديد السلالات الأكثر ملائمة لكل بيئة إختبار.

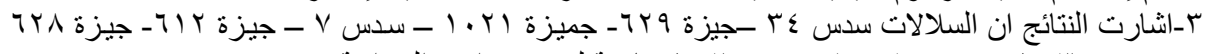

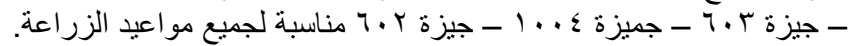
ع - لقد اظهر تحليل AMMI ان أفضل ميعاد زراعة هو الميعاد الثانى الذى أعطى أعلى أول مكون رئيسى

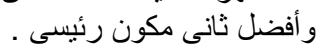

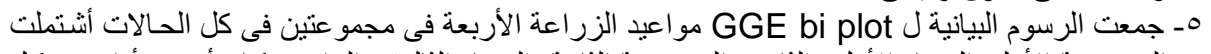

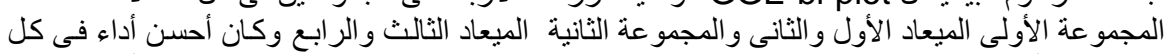

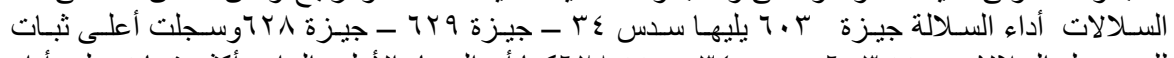

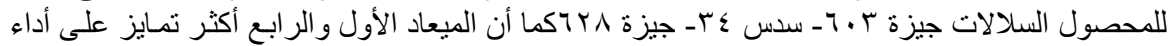

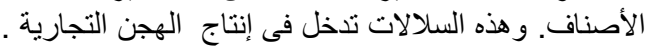

